\title{
Editorial
}

\section{Pulmonary Fibrosis as Sequelae of Covid-19 Pneumonia}

\author{
Md. Azizul Haque ${ }^{1}$
}

The extent, severity and impact of long-term respiratory complications of covid-19 is not yet fully explored, but early data shows that many survivors persistent respiratory symptoms months after their initial illness. Potential post-covid-19 problems include chronic cough, fibrotic lung disease, bronchiectasis and pulmonary vascular disease. ${ }^{1}$

The evidence for these sequelae is derived from our current experience of covid-19 along with extrapolation of past data from SARS-CoV 1 outbreak and patients with ARDS due aetiology other than covid. ${ }^{2}$ It is reported that approximately $30 \%$ of people with SARS or MERS had longterm respiratory abnormalities after their acute illness. $^{2}$

In the Beijing study on SARS, 71 patients underwent serial computed tomography from 2003 to 2018. Parenchymal abnormalities (ground glass opacity and "cord-like consolidation") were noted in 27 patients (38\%), but after 12 months these generally occupied less than $10 \%$ of the lung. Investigators noted that the degree of functional impairment was disproportionate to the extent of physiological impairment and concluded that muscle weakness due to deconditioning was a contributing factor. ${ }^{3,4}$

Lung injury in covid-19 is mostly a result of maladaptive immune responses leading to excessive cytokine release. Results from a recent Greek study reveal that patients with covid-19, who developed severe respiratory failure, display hyper-inflammatory responses with features of either immune dysregulation or macrophage activation syndrome. ${ }^{5}$ The pathogenesis of postcovid pulmonary fibrosis includes dysregulated release of matrix metalloproteinases during the inflammatory phase of adult respiratory distress syndrome (ARDS) causing epithelial and endothelial injury with unchecked fibroproliferation. There is also a vascular dysfunction which is a key component of the switch from ARDS to fibrosis, with vascular endothelial growth factor and cytokines such as interleukin-6 and tumor necrosis factor alpha being implicated. ${ }^{6}$

Current data shows that about $40 \%$ of patients with covid-19 develop ARDS, and 20\% of ARDS cases are severe. It is still too early to comment about the exact prevalence of post-covid-19 fibrosis, but early analysis from patients with covid-19 on hospital discharge suggests that more than a third of recovered patients develop fibrotic abnormalities. Additionally, $47 \%$ of patients had impaired diffusing capacity of the lungs for carbon monoxide (DLCO) and 25\% had reduced total lung capacity (TLC). This seemed even worse in patients with severe disease. ${ }^{7,8}$ In a study of 62 patients by Zhou et al., pulmonary fibrotic changes were noted in 21 (33.9\%) patients, and it was more likely to occur in advanced-phase disease (8-14 days after the onset of symptoms) than early phase of the disease ( $\leq 7$ days after the onset of symptoms). ${ }^{9}$

Long-term outcomes of covid-19 vary from person to person, as many patients have various degrees of pre-existing comorbidities. As pulmonary

${ }^{1}$ Associate Professor, Department of Medicine, Rajshahi Medical College, Bangladesh. 
fibrosis is a sequelae of pulmonary inflammation in covid-19, treatment often targets inflammation. High dose steroids were given routinely prescribed to many patients with SARS CoV-1, and this might partly explain the limited incidence of fibrosis observed. ${ }^{4}$ The benefit of dexamethasone in severe covid-19 has recently been established, but treatment did not improve acute outcomes among patients with milder disease, but it is still too early to comment on the long-term effects of steroid on post-covid-19 pulmonary fibrosis. ${ }^{10}$ Pirfenidone and nintedanib are the two approved antifibrotic drugs that are both effective in attenuating the rate of lung function decline and are widely considered to improve life expectancy in patients with IPF. ${ }^{11,12}$ The use of antifibrotic therapies could also be under consideration in post-covid pulmonary fibrotic disease, but it is still too early to make a firm recommendation. The current literature suggests that, any potential antifibrotic intervention should be considered within the first week of ARDS onset so as to be more effective. ${ }^{13}$

British thoracic society has published guidance for respiratory follow-up of covid-19 pneumonia. For all patients with covid-19 pneumonia who required admission in ICU or HDU, follow-up clinical respiratory assessment should be done 4-6 weeks after discharge, and a follow-up chest X-ray (CXR) should be done at 12 weeks. If there are no abnormalities in CXR, no further evaluation is needed. If CXR is not cleared satisfactorily, full pulmonary function evaluation should be done. If there are persistent CXR changes and/or evidence of physiological impairment is found, a precontrast high resolution volumetric CT and a CT pulmonary angiogram (CTPA) should be done to assess for the presence of both interstitial lung disease and pulmonary emboli. For patients with a mild to moderate clinico-radiological diagnosis of covid-19 pneumonia not requiring ICU or HDU care, respiratory follow up at 4-6 weeks is not needed; patients should be evaluated by CXR at 12 weeks and thereafter managed accordingly. ${ }^{14}$

Pulmonary fibrosis as sequelae of covid-19 pneumonia is a cause for global concern. Further research is needed to develop optimum management protocol and to predict the long-term outcome.

\section{References}

1. Asthma UK, British Lung Foundation. Post-covid hub. https://www.post covid.org.uk/get-support/

2. NHS England. Aftercare needs of inpatients recovering from COVID-19. Jun 2020. https://www.england.nhs.uk/coronavirus/publication lafter-care-needs-of-inpatients-recoveringfromcovid-19/

3. Ngai JC, Ko FW, Ng SS, To KW, Tong M, Hui DS. The long-term impact of severe acute respiratory syndrome on pulmonary function, exercise capacity and health status. Respirology. 2010; 15:543-50. doi: 10.1111/j.1440-1843.2010.01720.

4. Zhang $\mathrm{P}$, Li J, Liu H, etal. Long-term bone and lung consequences associated with hospital-acquired severe acute respiratory syndrome: a 15-year follow-up from a prospective cohort study. Bone Res. 2020; 8:8. doi: 10.1038/s41413-020-0084-5

5. Giamarellos-Bourboulis EJ, Netea MG, Rovina N, Akinosoglou K, Akinosoglou K, Antoniadou A, Antonakos $\mathrm{N}$ et al: Complex immune dysregulation in COVID-19 patients with severe respiratory failure. Cell Host Microbe. 2020; 27: 992-1000.

6. Burnham EL, Janssen WJ, Riches DW, Moss M, Downey GP. The fibroproliferative response in acute respiratory distress syndrome: mechanisms and clinical significance. Eur Respir J. 2014; 43:276-85.

7. Wu C, Chen X, Cai Y, Xia J, Zhou X, Xu S, Huang $\mathrm{H}$, Zhang $\mathrm{L}$, Zhou $\mathrm{X}$, Du C, et al: Risk factors associated with acute respiratory distress syndrome and death in patients with coronavirus disease 2019 pneumonia in Wuhan, China. JAMA Intern Med. 2020; 180(7):934-943. doi: 10. 1001/ jamainternmed. 2020.0994

8. Mo X, Jian W, Su Z, Chen M, Peng H, Peng P, Lei C, Chen R, Zhong $\mathrm{N}$ and Li S: Abnormal pulmonary function in COVID-19 patients at time of hospital discharge. Eur Respir J. 2020 Jun;55(6): 2001217.

9. S. Zhou, Y. Wang, T. Zhu, and L. Xia, CT features of coronavirus disease 2019 (COVID-19) pneumonia in 62 patients in Wuhan, China. Am J Roentgenol. 2020 Jun;214(6):1287-1294.

10. Dexamethasone in Hospitalized Patients with Covid-19 - Preliminary Report. The RECOVERY Collaborative Group N Engl J Med. 2020 Jul 17: NEJMoa2021436. Published online 2020 Jul 17. doi: 10.1056/NEJMoa2021436 
11. Margaritopoulos GA, Trachalaki A, Wells AU, Vasarmidi E, Bibaki E, Papastratigakis $G$ et al. Pirfenidone improves survival in IPF: Results from a real-life study. BMC Pulm Med. 2018;18:177,

12. Antoniou K, Markopoulou K, Tzouvelekis A, Trachalaki A, Vasarmidi E, Organtzis $\mathrm{J}$ et al: Efficacy and safety of nintedanib in a Greek multicentre idiopathic pulmonary fibrosis registry: A retrospective, observational, cohort study. ERJ Open Res. 2020; 6:00172-02019.
13. Eirini vasarmidi, Eliza Tsitoura, Demetrios A. Spandidos, Antoniou KM. Pulmonary fibrosis in the aftermath of covid-19 era (Review). Experimental and Therapeutic Medicine. 2020; 20:2557-2560.

14. British Thoracic Society. Guidance on respiratory follow up of patients with a clinico-radiological diagnosis of covid-19 pneumonia. 2020. www.britthoracic.org.uk

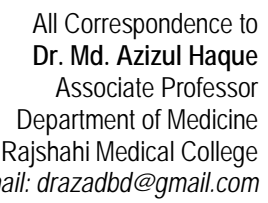

All Correspondence to Associate Professor Department of Medicine Email: drazadbd@gmail.com 